\title{
DETERMINAÇÃO SIMULTÂNEA DE RESÍDUOS DE SULFAMETOXAZOL E TRIMETOPRIMA EM SUPERFÍCIES DE EQUIPAMENTOS DE PRODUÇÃO
}

\author{
Roberto C. Coutinho e Elder T. Barbosa \\ Neo Química,VPR1, Quadra 2A, Módulo 4, DAIA, 75000-000 Anápolis - GO, Brasil \\ Marcelo M. Sena* e Caridad Noda Pérez \\ Unidade Universitária de Ciências Exatas e Tecnológicas, Universidade Estadual de Goiás, CP 459, 75000-000 Anápolis - GO, Brasil
}

Recebido em 22/1/09; aceito em 28/4/09; publicado na web em 30/9/09

\begin{abstract}
SIMULTANEOUS DETERMINATION OF SULFAMETHOXAZOLE AND TRIMETHOPRIM RESIDUES ON MANUFACTURING EQUIPMENT SURFACES. A cleaning validation method was developed and validated, based on swabbing sampling and simultaneous chromatographic determination of sulfamethoxazole (SMX) and trimethoprim (TMP) residues. The method presented limits of detection of $0.06 \mu \mathrm{g} \mathrm{mL}^{-1}$ for SMX and $0.09 \mu \mathrm{g} \mathrm{mL} \mathrm{L}^{-1}$ for TMP. It was considered selective, precise, accurate and robust according to the guidelines from ANVISA, the Brazilian regulatory agency, and International Conference on Harmonization. Mean swab recovery factors of $98.5 \%$ for SMX and $97.7 \%$ for TMP were obtained for spiked stainless steel plates. The method was successfully applied to the assay of actual swab samples collected from eleven points on an equipment surface.
\end{abstract}

Keywords: HPLC; cleaning validation; swabbing recovery.

\section{INTRODUÇÃO}

O primeiro caso de contaminação cruzada na indústria farmacêutica relatado na literatura científica ocorreu nos anos 60 , quando graves e inesperados efeitos colaterais foram observados devido à contaminação de isoniazida (hidrazida do ácido isonicotínico) com dietilestilbestrol. ${ }^{1}$ Nos últimos anos, vários produtos farmacêuticos foram retirados do mercado devido à contaminação cruzada com outros princípios ativos ou substâncias químicas. ${ }^{2}$ Em consequência disto, a validação de limpeza tornou-se um aspecto crítico a ser enfrentado pela indústria farmacêutica. O seu objetivo é provar, através de um método analítico validado e suficientemente sensível, que o procedimento de limpeza de equipamentos e superfícies é eficiente na remoção de resíduos de princípios ativos, excipientes, produtos de degradação, substâncias usadas na limpeza e outros possíveis contaminantes, reduzindo assim o risco de contaminação cruzada na área de produção. Desse modo, é necessário tanto assegurar que os contaminantes sejam removidos do equipamento, quanto determinar o nível de consistência dessa remoção. Este assunto é parte integrante do conjunto de normas que compõem as boas práticas de fabricação (BPF) e a validação de limpeza deve seguir as resoluções dos organismos reguladores. ${ }^{3-5}$

Como os equipamentos são usados na produção de diversas formulações farmacêuticas, costuma-se definir um cenário de pior caso. ${ }^{6,7}$ Um produto de referência é escolhido como o pior caso para a validação de limpeza, levando em conta variáveis relacionadas à sua toxicidade e dificuldade de limpeza. Uma vez que o produto considerado o pior caso tenha sido escolhido, surge um outro aspecto importante que é a amostragem. Para isso, dois métodos são indicados: ${ }^{5}$ a amostragem direta da superfície, usando a técnica de swabbing (escovação) e, a amostragem indireta, baseada na análise das soluções usadas para enxaguar o equipamento. Na maior parte da literatura, a técnica de swabbing é a preferida, porque permite que resíduos secos e/ou insolúveis sejam mais eficientemente amostrados por remoção física e torna possível avaliar as áreas do equipamento mais difíceis de limpar.

\footnotetext{
*e-mail: marcsen@ueg.br
}

A crescente relevância da validação de limpeza pode ser constatada pelo aumento do número de artigos científicos tratando deste tema. A maioria destes aplicou técnicas cromatográficas, dentre elas CLAE ${ }^{8-13}$ cromatografia líquida de ultra eficiência (CLUE), ${ }^{14}$ cromatografia líquida acoplada à espectrometria de massas ${ }^{15}$ e cromatografia eletrocinética micelar (MEKC) ${ }_{1}^{16}$ para determinar resíduos de princípios ativos farmacêuticos, tais como anlodipina, ${ }^{8}$ azatioprina, ${ }^{9}$ lacidipina, ${ }^{10}$ derivados cortisônicos, ${ }^{11}$ lamivudina e zidovudina, ${ }^{12}$ pirimetamina e sulfadoxina, ${ }^{13}$ compostos esteroidais, ${ }^{14}$ cefmetazol e cefpodoxima proxetil, ${ }^{15}$ e cotrimetoxazol. ${ }^{16} \mathrm{Na}$ presente aplicação, a associação entre sulfametoxazol (SMX) e trimetoprima (TMP) foi selecionada para a validação de limpeza de um equipamento de produção. O SMX, ${ }^{17}$ N1-(5-metil-3-isoxazolil)sulfanilamida, é uma sulfonamida solúvel em acetona e metanol, e é usado primariamente no tratamento de infecções do trato urinário. Ele possui alta absorção e excreção, podendo causar cristaluria (presença de cristais na urina) em altos níveis de sua forma acetilada. A TMP, ${ }^{18}$ 2,4-diamino-5-(3,4,5-trimetoxibenzil)-pirimidina, é um pó cristalino amarelo claro, pouco solúvel em metanol e clorofórmio. Para indivíduos com deficiência de ácido fólico, tais como idosos e mulheres grávidas, a TMP apresenta algum risco de toxicidade. A associação entre SMX e TMP possui atividade antimicrobial eficiente, pois esses dois fármacos atuam respectivamente em estágios sequenciais da reação enzimática bacteriana, e é usada no tratamento de otites, bronquites, sinusites e pneumonias. ${ }^{19}$ Os métodos encontrados na literatura para a determinação simultânea de SMX e TMP em formulações farmacêuticas são baseados em CLAE, ${ }^{20-22}$ eletroforese capilar $^{23,24}$ e espectrofotometria com detecção multivariada..$^{25,26}$

O objetivo deste trabalho foi desenvolver e validar um método analítico para a validação de limpeza das superfícies de um equipamento de produção farmacêutica, através da determinação cromatográfica simultânea de resíduos de SMX e TMP e usando a técnica de swabbing para amostragem. Ao invés de usar metanol, como na maioria dos trabalhos com swabbing, optou-se por um solvente menos tóxico, uma mistura etanol-água. A metodologia analítica foi validada de acordo com as resoluções da Agência Nacional de Vigilância Sanitária (ANVISA), 3,27 que por sua vez se baseiam no documento Q2B do ICH (International Conference on Harmonization),${ }^{28}$ e foi aplicada na determinação dos resíduos em onze pontos de amostragem na superfície do equipamento. 


\section{PARTE EXPERIMENTAL}

\section{Reagentes}

O SMX e a TMP foram fornecidos pela Neo Química (Anápolis) e usados como padrões secundários (99,6 e 99,4\%, respectivamente), de acordo com a certificação da empresa. Foram utilizados etanol, acetonitrila e trietilamina, todos de grau cromatográfico (Tedia), hidróxido de sódio (J. T. Baker) e água deionizada (Milli-Q). A amostragem foi feita usando swabs (instrumentos de limpeza com uma haste flexível e uma extremidade absorvente) de poliéster TX714A Texwipe (Large Alpha ${ }^{\circledR}$ ).

\section{Equipamento e condições cromatográficas}

O sistema CLAE utilizado consistiu de um módulo de separação Waters Alliance 2695, com uma bomba quaternária de baixa pressão, um desgaseificador a vácuo em linha, um amostrador automático e um detector UV-Vis de arranjo de diodos modelo 2996. Para o controle do sistema cromatográfico e coleta dos dados foi usado o programa Waters Empower. Foi usada uma coluna C-18 (250 x 4,6 $\mathrm{mm})$ Waters Symmetry com partículas de $5 \mu \mathrm{m}$. A fase móvel foi uma mistura de água/acetenotrilina/trietilamina (77,7:22,2:0,1, v/v) com o pH ajustado em 5,9 (NaOH), em um fluxo de 2,5 $\mathrm{mL} \mathrm{min}^{-1}$. O volume de injeção foi de $20 \mu \mathrm{L}$ e a detecção no UV foi feita em 254 $\mathrm{nm}$. Todas as análises foram feitas em temperatura ambiente $\left(25^{\circ} \mathrm{C}\right)$.

\section{Preparação dos padrões de calibração}

As soluções estoque de SMX e TMP foram preparadas pesando-se exatamente $20 \mathrm{mg}$ de cada padrão, os quais foram transferidos para balões volumétricos de $200 \mathrm{~mL}$, respectivamente. Os padrões foram dissolvidos em mistura etanol/água (50:50, v/v), com o auxílio de um banho ultrassônico (15 min), e os balões foram completados com o mesmo solvente. A partir da diluição dessas soluções, foram preparadas sete soluções de calibração na faixa de 2,0 a $14,0 \mu \mathrm{g} \mathrm{mL}^{-1}$ para cada analito. As alíquotas dessas soluções padrão foram filtradas através de membranas de $0,45 \mu \mathrm{m}$ de porosidade.

\section{Amostragem}

A amostragem foi feita com swabs descartáveis de poliéster, previamente embebidos com uma mistura etanol/água (50:50, v/v). Placas de aço inoxidável de $100 \mathrm{~cm}^{2}$ foram contaminadas com 1,00 mL de soluções de SMX e TMP, respectivamente. As soluções colocadas na superfície de teste foram deixadas para evaporar. Cada superfície de placa foi limpa com um swab de várias maneiras (horizontalmente, verticalmente, de trás para frente e de frente para trás), começando das bordas para o centro. Na sequência, o $s w a b$ foi colocado em um tubo contendo $10,0 \mathrm{~mL}$ de etanol/água, o qual foi selado e colocado em um banho ultrassônico por 15 min. Alíquotas das soluções obtidas na recuperação das placas foram filtradas através de membranas de $0,45 \mu \mathrm{m}$ de porosidade e injetadas no cromatógrafo.

\section{Amostras reais coletadas de um equipamento de produção}

A metodologia de validação de limpeza foi aplicada a um granulador Collette (Niro Pharma, Wommelgem, Bélgica), que é um equipamento de aço inox automático usado na pulverização e mistura de substâncias sólidas em ambiente úmido, no setor de fabricação de sólidos. Após o processo de validação de limpeza, 11 pontos representativos de todo o equipamento foram amostrados por swabbing: 5 pontos nas hélices do misturador, 1 ponto na tampa superior, 2 pontos nas paredes, 1 ponto no fundo e 2 pontos na saída do equipamento por onde o produto é retirado. A amostragem foi feita como descrita na seção anterior. Três lotes diferentes foram analisados.

\section{RESULTADOS E DISCUSSÃO}

\section{Escolha do pior caso}

Dez formulações farmacêuticas diferentes são produzidas nesse equipamento: aciclovir, atenolol, cloranfenicol, clorpropamida, dipirona, furosemida, ibuprofeno, metildopa, metronidazol e a associação SMX-TMP. Como não é prático validar o processo de limpeza para todos esses produtos, segue-se a recomendação de escolher o pior caso. ${ }^{6,713}$ Então, um índice de pior caso (WCI, worst case index) foi calculado para cada produto, o qual se baseia na sua solubilidade, na sua toxicidade, no nível de dificuldade para sua limpeza no equipamento e no tamanho do seu lote. ${ }^{6}$ A associação SMX-TMP apresentou o maior valor de WCI e por isso foi escolhida para a validação de limpeza.

\section{Limites de aceitação}

O estabelecimento dos limites de aceitação dos resíduos é necessário para a definição da faixa de aplicação do método analítico. Para determinar os limites de aceitação do(s) fármaco(s), cuja metodologia será validada, primeiramente considera-se o limite de sua aceitação no produto produzido em sequência no equipamento $\left(\mathrm{L}_{1}\right.$, limite no produto subsequente). Depois, o(s) limite(s) por área superficial do equipamento a ser limpo $\left(\mathrm{L}_{2}\right)$ é(são) estimado(s) e, finalmente, o limite do resíduo na amostra analisada $\left(\mathrm{L}_{3}\right)$ é calculado. ${ }^{29}$ Esses limites são calculados de acordo com as seguintes equações:

$\mathrm{L}_{1}=0,001 \times(\mathrm{DT} / \mathrm{MDD})$

$\mathrm{L}_{2}=\mathrm{L}_{1} \times(\mathrm{LS} / \mathrm{AT})$

$\mathrm{L}_{3}=\mathrm{L}_{2} \times(\mathrm{AS} / \mathrm{VS})$

Para o presente caso, a menor dose terapêutica (DT) foi estimada como 1600 mg para o SMX e 320 mg para o TMP; a maior dose diária (MDD) do produto subsequente (escolhido como o princípio ativo com o menor tamanho de lote pela máxima dose diária, neste caso o ibuprofeno) foi 5,03 g; o tamanho do lote subsequente (LS) foi 79447 g; a superfície da área total da linha de produção do equipamento (AT) foi 7,926 x $10^{5} \mathrm{~cm}^{2}$; a área superficial (AS) foi $100 \mathrm{~cm}^{2}$; o volume de solvente usado (VS) foi 10,0 mL. Com esses dados, $\mathrm{L}_{1}$ foi estimado como $318,3 \mu \mathrm{g} \mathrm{g}^{-1}$ para o SMX e $63,7 \mu \mathrm{g} \mathrm{g}^{-1}$ para o TMP. Porém, estes valores estão acima do mínimo estabelecido pela FDA, ${ }^{5}$ que é de $10,0 \mu \mathrm{g} \mathrm{g}^{-1}$, o que então levou à adoção deste último valor como limite para ambos os analitos neste trabalho. Como consequência, $\mathrm{L}_{2}$ e $\mathrm{L}_{3}$ foram calculados como $1,002 \mu \mathrm{g} \mathrm{cm}^{-2}$ e $10,02 \mu \mathrm{g} \mathrm{mL}^{-1}$, respectivamente. Portanto, um limite de aceitação de $10,0 \mu \mathrm{g} \mathrm{mL}^{-1}$ para ambos os analitos foi adotado no processo de limpeza e a faixa do método analítico foi estabelecida de acordo com isto.

\section{Validação do método analítico}

A validação do método foi realizada através da estimativa das seguintes figuras de mérito: fator de recuperação, seletividade, linearidade, precisão, exatidão, limites de detecção e quantificação e robustez.

Os fatores de recuperação para a amostragem por swabbing foram estimados em placas de aço inox de $100 \mathrm{~cm}^{2}$ contaminadas com 100,0 $\mu \mathrm{g}$ de SMX e 100,0 $\mu \mathrm{g}$ de TMP. Essas quantidades foram extraídas 
em 10,0 mL de solvente, correspondendo a concentrações finais de $10,0 \mu \mathrm{g} \mathrm{mL}^{-1}$. Para 6 amostras, o fator de recuperação da extração variou de 97,0 a 99,0\%, com média de 98,5\%, para o SMX e de 96,6 a $98,4 \%$, com média de $97,7 \%$, para a TMP.

Um cromatograma típico para os padrões de SMX e TMP é mostrado na Figura 1. O método foi considerado seletivo, porque nenhuma interferência foi observada nos tempos de retenção dos analitos na análise dos excipientes e do branco das amostras de extração (swab).

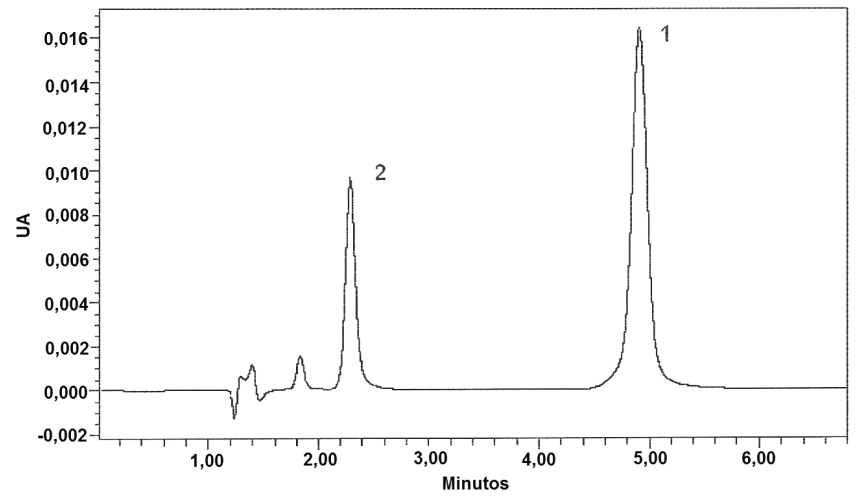

Figura 1. Cromatograma obtido para os padrões de SMX (1) e TMP (2), ambos na concentração de $10,0 \mu \mathrm{g} m L^{-1}$

A linearidade do método foi avaliada pela análise em sete níveis de concentração igualmente espaçados de 2,0 a $14,0 \mu \mathrm{g} \mathrm{mL}^{-1}$ para cada analito, com cada nível em triplicata. As seguintes equações de regressão foram obtidas para as retas da área do pico versus a concentração do analito $\left(\mu \mathrm{g} \mathrm{mL}^{-1}\right): \mathrm{y}=19278,7 \mathrm{x}-4655,7(\mathrm{r}=0,9995)$ para SMX e $y=7304,7 x-402,3(r=0,9998)$ para TMP. Os coeficientes de correlação (r) estimados demonstraram a boa linearidade do método.

A precisão foi avaliada nos níveis de repetibilidade e precisão intermediária. A repetibilidade (intradia) foi estimada pelo mesmo analista para 6 replicatas de amostras extraídas por swabbing na concentração de $10,0 \mu \mathrm{g} \mathrm{mL}^{-1}$ para cada analito. Todos os resultados foram corrigidos pelos fatores de recuperação. Os valores de desvio padrão relativo (DPR) foram $0,63 \%$ para SMX e 0,82\% para TMP. A precisão intermediária foi estimada para 6 replicatas no mesmo nível de concentração em 2 dias, com analistas diferentes e colunas cromatográficas de fabricantes diferentes a cada dia. Os valores de DPR para os resultados entre os 2 dias foram $0,82 \%$ para SMX e $4,70 \%$ para TMP, ambos abaixo de $5 \%$, o valor máximo permitido pela ANVISA. ${ }^{27}$

A exatidão foi avaliada pela comparação da quantidade de analito prevista na recuperação por swabbing com a quantidade conhecida de analito usada na contaminação de placas de aço, em três níveis de concentração $\left(8,0,10,0\right.$ e 12,0 $\left.\mu \mathrm{g} \mathrm{mL}^{-1}\right)$, com 9 replicatas para cada nível. Os resultados foram corrigidos pelos fatores de recuperação e são mostrados na Tabela 1. O grau de recuperação variou entre 98,0 e $100,0 \%$ para SMX e entre 92,5 e $106,7 \%$ para TMP.
Os limites de detecção (LD) e quantificação (LQ) foram estimados baseados na inclinação e nos desvios padrão do intercepto em y das retas das três curvas de calibração. ${ }^{27,28}$ Os valores de LD de 0,06 e $0,09 \mu \mathrm{g} \mathrm{mL}^{-1}$ e de LQ de 0,19 e $0,30 \mu \mathrm{g} \mathrm{mL}^{-1}$ foram estimados para SMX e para TMP, respectivamente. Estes valores teóricos foram comprovados visualmente pela observação dos cromatogramas obtidos para injeções feitas nos respectivos níveis de concentração.

O método foi considerado robusto em relação a pequenas e deliberadas variações de $\mathrm{pH}(5,7$ e 6,1$)$ e composição da fase móvel, não apresentando mudança significativa nos parâmetros cromatográficos (tempo de retenção, número de pratos teóricos e resolução dos picos). Além disso, a estabilidade das soluções analíticas foi estudada até $21 \mathrm{~h}$. Os desvios observados nas áreas dos picos foram de até $0,80 \%$ para SMX e 1,41\% para TMP nas soluções dos padrões; e 1,58\% para SMX e 1,08\% para TMP, nas amostras de soluções extraídas de placas de aço. Esses resultados indicam a robustez do método.

\section{Determinação das amostras coletadas nas superfícies de um equipamento de produção}

O método, depois de validado, pode ser aplicado na validação de limpeza de equipamentos de produção. Três lotes de produção da associação SMX-TMP no granulador Collette foram analisados. Após cada lote, o processo de limpeza foi aplicado ao equipamento. Então, amostras reais foram coletadas por swabbing em 11 pontos representativos de sua superfície interna e analisadas pelo método validado. Todos os resultados para a determinação de resíduos de SMX e TMP ficaram abaixo dos respectivos valores de LD. Portanto, foi certificada a não ocorrência de contaminação no equipamento após a produção desses lotes.

\section{CONCLUSÕES}

Este trabalho propôs uma metodologia analítica completa para a validação de limpeza de resíduos de SMX e TMP em superfícies de equipamentos de produção farmacêutica, incluindo a extração por swabbing usando uma mistura não tóxica (etanol-água) como solvente e o método cromatográfico para a determinação simultânea desses dois antibióticos. O método foi validado de acordo com as resoluções da ANVISA $^{3,27}$ e considerado rápido (tempo de corrida cromatográfica de $6 \mathrm{~min}$ ), seletivo, sensível, preciso, exato e robusto, além de linear na faixa de interesse de 2,0 a $14,0 \mu \mathrm{g} \mathrm{mL}^{-1}$. Adicionalmente, a estabilidade das soluções analíticas sobre a superfície de aço inox em até $21 \mathrm{~h}$ foi verificada, o que é importante na prevenção de que os resíduos sejam sobre-estimados, já que a degradação dos analitos pode levar à detecção de quantidades menores do que as realmente existentes. Este trabalho atendeu a uma demanda específica de uma indústria farmacêutica, representando um exemplo de aproximação entre uma universidade pública e o setor produtivo.

Tabela 1. Estimativa da exatidão para a determinação simultânea de SMX e TMP

\begin{tabular}{|c|c|c|c|c|c|}
\hline SMX & & & TMP & & \\
\hline $\begin{array}{l}\text { Quantidade adicionada } \\
\left(\mu \mathrm{g} \mathrm{mL}^{-1}\right)\end{array}$ & $\begin{array}{l}\text { Quantidade prevista } \\
\qquad(\mu \mathrm{g} \mathrm{mL}-1)\end{array}$ & $\begin{array}{c}\text { Grau de recuperação } \\
(\%)\end{array}$ & $\begin{array}{l}\text { Quantidade adicionada } \\
\qquad\left(\mu \mathrm{g} \mathrm{mL} \mathrm{mL}^{-1}\right)\end{array}$ & $\begin{array}{l}\text { Quantidade prevista } \\
\qquad(\mu \mathrm{g} \mathrm{mL}-1)\end{array}$ & $\begin{array}{c}\text { Grau de recuperação } \\
(\%)\end{array}$ \\
\hline 8,0 & 8,0 & 100,0 & 8,0 & 7,4 & 92,5 \\
\hline 10,0 & 9,8 & 98,0 & 10,0 & 9,9 & 99,0 \\
\hline 12,0 & 11,9 & 99,2 & 12,0 & 12,8 & 106,7 \\
\hline
\end{tabular}

a Valores médios de nove determinações 


\section{REFERÊNCIAS}

1. Weber, W. W.; Duffy, M. P.; Thom, J. V.; Grossman, M.; Sax, J.; Chan, J. J.; N. Engl. J. Med. 1963, 268, 411.

2. Prosek, M.; Krizman, M.; Kovac, M.; J. Pharm. Biomed. Anal. 2005, 38, 508.

3. Agência Nacional de Vigilância Sanitária; Resolução RDC 210, de 04/08/2003.

4. International Conference on Harmonization Tripartite Guideline; $Q 7 A$ : Manufacturing Practice Guideline for Active Pharmaceutical Ingredients, US Food and Drug Administration, 2000.

5. United States Food and Drug Administration; Guide to Inspections Validation of Cleaning Processes, Office of Regulatory Affairs: Washington, 1993.

6. Rodricks, J. V; Haz. Waste Haz. Mat. 1984, 1, 333.

7. Mazonakis, N. E.; Karathanassi, P. H.; Panagiotopoulos, D. P.; Hamosfakidi, P. G.; Melissos, D. A.; Anal. Chim. Acta 2002, 467, 261

8. Klinkenberg, R.; Streel, B.; Ceccato, A.; J. Pharm. Biomed. Anal. 2003, 32,345 .

9. Fazio, T. T.; Singh, A. K.; Kedor-Hackmann, E. R. M.; Santoro, M. I. R M.; J. Pharm. Biomed. Anal. 2007, 43, 1495.

10. Nozal, M. J.; Bernal, J. L.; Jimenez, J. J.; Martin, M. T.; Diez, F. J.; J. Chromatogr., A 2004, 1024, 115.

11. Hamdan, I. I.; Qurani, H.; J. Liq. Chrom. Rel. Technol. 2009, 32, 449.

12. Santoro, M. I. R. M.; Fazio, T. T.; Singh, A. K.; Kedor-Hackmann, E. R. M.; J. AOAC Int. 2007, 90, 715.

13. Boca, M. B.; Apostolides, Z.; Pretorius, E.; J. Pharm. Biomed. Anal. 2005, 37, 461 .
14. Fekete, S.; Fekete, J.; Ganzler, K.; J. Pharm. Biomed. Anal. 2009, 49, 833.

15. Fukutsu, N.; Sakamaki, Y.; Kawasaki, T.; Saito, K.; Nakazawa, H.; J. Pharm. Biomed. Anal. 2006, 41, 1243.

16. Boca, M. B.; Pretorius, E.; Kgaje, C.; Apostolides, Z.; J. Pharm. Biomed. Anal. 2008, 46, 631.

17. Rudy, B. C.; Senkowski, B. Z. Em Analytical Profiles of Drug Substances 2; Florey, K., ed.; Academic Press: New York, 1973, p. 467.

18. Manius, G. J. Em Analytical Profiles of Drug Substances 7; Florey, K., ed.; Academic Press: New York, 1978, p. 445.

19. Gilman, A. G.; Hardman, J. G.; Limbird, L. E.; As Bases Farmacológicas da Terapêutica, $10^{\mathrm{a}}$ ed., Mac Graw Hill: Rio de Janeiro, 2003.

20. Akay, C.; Ozkan, S. A.; J. Pharm. Biomed. Anal. 2002, 30, 1207.

21. Dinc, E.; Bilgili, A.; Hanedan, B.; Pharmazie 2007, 62, 179.

22. Farmacopéia Brasileira, 4ª ed., Atheneu Ed.: São Paulo, 2004.

23. Nevado, J. J. B.; Penalvo, G. C.; Bernardo, F. J. G.; J. Chromatogr., A 2001, 918, 205

24. Cao, X. D.; Fang, Q.; Fang, Z. L.; Anal. Chim. Acta 2004, 513, 473.

25. Hassouna, M. E. M.; Anal. Lett. 1997, 30, 2341.

26. Lopez Martinez, L.; Lopez de Alba, P. L.; de Leon Rodriguez, L. M.; Yepez Murrieta, M. L.; J. Pharm. Biomed. Anal. 2002, 30, 77.

27. Agência Nacional de Vigilância Sanitária; Guia para Validação de Métodos Analíticos e Bioanalíticos, Resolução RE 899, de 29/05/2003.

28. International Conference on Harmonization Tripartite Guideline; $Q 2 B$ : Validation of Analytical Procedures: Methodology, US Food and Drug Administration, 1997.

29. LeBlanc, D. A.; Pharm. Tech. 2000, 24, 160. 